\title{
ON TESTABILITY OF COMPLEMENTARITY IN MODELS WITH MULTIPLE EQUILIBRIA
}

\author{
YOSHIYASU RAI AND TAISUKE OTSU
}

\begin{abstract}
This paper revisits testability of complementarity in economic models with multiple equilibria studied by Echenique and Komunjer (2009). We find that Echenique and Komunjer's (2009) testable implications on extreme quantiles can be implied by a weaker version of their tail condition without complementarity, and on the other hand, a slightly stronger version of complementarity implies their testable implications without the tail condition.
\end{abstract}

Keywords: Complementarity; Testability; Quantile.

JEL Code: C10

\section{MAIN RESUlT}

We first introduce the setup and main result of Echenique and Komunjer (2009) (hereafter, EK). Our notation closely follows EK's. Consider a structural equation:

$$
r(Y, X)=U
$$

where $Y \in \mathbb{R}$ is a dependent variable, $X \in \mathcal{X} \subseteq \mathbb{R}$ is an explanatory variable, $U \in \mathbb{R}$ is a disturbance term, and $r: \mathbb{R} \times \mathcal{X} \rightarrow \mathbb{R}$ is a function implied by economic theory. We observe $X$ and $Y$ but do not observe $U$. EK studied testability of this model when there are complementarities between $X$ and $Y$ without assuming a parametric functional form of $r$, dependence structure between $X$ and $U$, and specific equilibrium selection rule. Let $\mathcal{E}_{x u}=\{y \in \mathbb{R}|| r(y, x)=u\}$ be the equilibrium set for given $x$ and $u, F_{U \mid X=x}$ be the conditional distribution of $U$ given $X=x$ (which is assumed to have a strictly positive density), and $\bar{F}_{Y \mid X=x}(y)=1-F_{Y \mid X=x}(y)$, where $F_{Y \mid X=x}$ is the conditional distribution of $Y$ given $X=x$.

By an innovative argument to focus on the largest or smallest equilibrium and to apply a change of variable technique, EK obtained the following result (the comments in the parentheses are added by the authors).

Assumption S1. (i) The function $r: \mathbb{R} \times \mathcal{X} \rightarrow \mathbb{R}$ is continuous (on $y \in \mathbb{R}$ for each given $X \in \mathcal{X}$ ); (ii) for any $x \in \mathcal{X}, \lim _{y \rightarrow-\infty} r(y, x)=+\infty$ and $\lim _{y \rightarrow+\infty} r(y, x)=-\infty$; (iii) for any $(x, u) \in \mathcal{X} \times \mathbb{R}, \mathcal{E}_{x u}$ is a finite set. We write $\mathcal{E}_{x u}=\left\{\xi_{1 x u}, \ldots, \xi_{n_{x} x u}\right\}\left(\xi_{1 x u} \leq \cdots \leq \xi_{n_{x} x u}\right)$ with $n_{x}=\operatorname{Card}\left(\mathcal{E}_{x u}\right)$ (which is finite and does not depend on $u$ ). (Also, the selection rule $\mathcal{P}_{x u}$, a probability distribution over $\mathcal{E}_{x u}$, assigns probabilities $\left\{\pi_{1 x}, \ldots, \pi_{n_{x} x}\right\}$ to $\left\{\xi_{1 x u}, \ldots, \xi_{n_{x} x u}\right\}$ for all $u \in \mathbb{R}$ with $\pi_{1 x}>0$ and $\pi_{n_{x} x}>0$.) 
Assumption S2. $r(y, x)$ is monotone increasing in $x$ on $\mathbb{R}$.

Assumption S3. (For any $x_{1}, x_{2} \in \mathcal{X}$ with $x_{1}<x_{2}$ ) (i) $\lim _{y \rightarrow+\infty}\left[r\left(y, x_{1}\right) / r\left(y, x_{2}\right)\right]=\lambda$ with $\lambda>1$; (ii) for any $\lambda>1$, $\lim _{u \rightarrow-\infty} F_{U \mid X=x_{1}}(\lambda u) / F_{U \mid X=x_{1}}(u)=0$; (iii) $F_{U \mid X=x_{1}}(u) / F_{U \mid X=x_{2}}(u)$ is bounded as $u \rightarrow-\infty$.

Theorem. (Echenique-Komunjer) Assume S1, S2, and S3 hold. Fix a selection rule $\mathcal{P}_{X U}$. For any $x_{1}, x_{2} \in \mathcal{X}$ with $x_{1} \leq x_{2}$, there exists $\bar{y} \in \mathbb{R}$ such that for all $y \geq \bar{y}, \bar{F}_{Y \mid X=x_{1}}(y) \leq$ $\bar{F}_{Y \mid X=x_{2}}(y)$.

EK argued that the above monotonicity in $x$ on the tail of $\bar{F}_{Y \mid X=x}$ is a testable implication of Assumption S2, complementarities between $y$ and $x$. By a close inspection of the proof of EK, we find that the conclusion of Theorem 1 can be derived without S2 and indeed S3 (i), which guarantees complementarities in the tail, is sufficient. We introduce the following weaker version of S3 (i).

Assumption S3. (i') $\lim _{y \rightarrow+\infty} \inf _{y^{\prime} \geq y}\left[r\left(y^{\prime}, x_{1}\right) / r\left(y^{\prime}, x_{2}\right)\right]=\lambda$ with $\lambda>1$.

Our findings are summarized as follows.

Proposition. Fix a selection rule $\mathcal{P}_{X U}$ and $x_{1}, x_{2} \in \mathcal{X}$ with $x_{1}<x_{2}$.

(i) Under $S 1$ and $S 3$ (i') and (ii)-(iii), the conclusion of Theorem 1 holds true.

(ii) Under $S 1$ and $S 3$, the conclusion of Theorem 1 holds true.

(iii) Under S1, S2, and S3 (iii), $\lim _{y \rightarrow+\infty} \bar{F}_{Y \mid X=x_{1}}(y) / \bar{F}_{Y \mid X=x_{2}}(y)$ is bounded.

(iv) Under $S 1, S 3\left(i^{\prime}\right)$ is neither necessary nor sufficient for S2.

(v) Under S1, S3 ( $\left.i^{\prime}\right)$ is sufficient but not necessary for complementarity at extremes (i.e., there exists $\bar{y} \in \mathbb{R}$ such that $r\left(y, x_{1}\right)<r\left(y, x_{2}\right)$ for all $\left.y \geq \bar{y}\right)$.

(vi) The conclusion of Theorem 1, S1, and S3 (ii)-(iii) are not sufficient for complementarity at extremes.

Proof of (i). Let $r^{\mathrm{e}}(y, x)$ be a nonincreasing upper envelope of $r(y, x)$ on $\left[y_{0},+\infty\right)$ for a given $y_{0} \in \mathbb{R}$, i.e., $r^{\mathrm{e}}(y, x)=\inf \left\{q(y): q\right.$ is nonincreasing on $\left[y_{0},+\infty\right)$ and $q(y) \geq r(y, x)$ for all $\left.\left[y_{0},+\infty\right)\right\}$. We proceed in three steps.

Step 1: Show that for any $\lambda_{1} \in(1, \lambda)$, there exists $\bar{y} \in \mathbb{R}$ such that $r\left(y, x_{1}\right) \leq \lambda_{1} r\left(y, x_{2}\right)$ for all $y \geq \bar{y}$. Pick any $\lambda_{1} \in(1, \lambda)$. By S3 (i'), there exists $y^{*} \in \mathbb{R}$ such that

$$
\inf _{y \geq y^{*}}\left[r\left(y, x_{1}\right) / r\left(y, x_{2}\right)\right] \geq \lambda_{1}
$$

for all $y \geq y^{*}$. Moreover, by S1 (ii), there exists $y^{\prime} \in \mathbb{R}$ such that $r\left(y, x_{2}\right) \leq-1$ for all $y \geq y^{\prime}$. Thus, by taking $\bar{y}=\max \left\{y^{*}, y^{\prime}\right\}$, we have $r\left(y, x_{1}\right) \leq \lambda_{1} r\left(y, x_{2}\right)$ for all $y \geq \bar{y}$. Thus, we obtain the conclusion of this step. 
Step 2: Show that there exists $\tilde{y} \in \mathbb{R}$ such that $r^{\mathrm{e}}\left(y, x_{1}\right) \leq \lambda_{1} r^{\mathrm{e}}\left(y, x_{2}\right)$ for all $y \geq \tilde{y}$. From Step 1 , take $\bar{y} \in \mathbb{R}$ such that $r\left(y, x_{1}\right) \leq \lambda_{1} r\left(y, x_{2}\right)$ for all $y \geq \bar{y}$. By taking $\tilde{y}=\bar{y}$ and letting $r^{\mathrm{e}}\left(y, x_{2}\right)$ be a nonincreasing upper envelope for $\tilde{y}$, we have $r\left(y, x_{1}\right) \leq \lambda_{1} r\left(y, x_{2}\right) \leq \lambda_{1} r^{\mathrm{e}}\left(y, x_{2}\right)$ for all $y \geq \tilde{y}$. Now from the definition of $r^{\mathrm{e}}(y, x)$,

$$
\begin{aligned}
r^{\mathrm{e}}\left(y, x_{1}\right) & =\inf \left\{q(y): q \text { is nonincreasing on }[\tilde{y},+\infty) \text { and } q(y) \geq r\left(y, x_{1}\right) \text { for all }[\tilde{y},+\infty)\right\} \\
& \leq \lambda r^{\mathrm{e}}\left(y, x_{2}\right)
\end{aligned}
$$

for all $y \geq \tilde{y}$. Thus, we obtain the conclusion of this step.

Step 3: Show $\lim _{y \rightarrow+\infty} \frac{\bar{F}_{Y \mid X=x_{1}}(y)}{\bar{F}_{Y \mid X=x_{2}}(y)}=0$. By Proposition 1 and Lemma 1 of EK, ${ }^{1}$ there exists $y^{\prime} \in \mathbb{R}$ such that

$$
\frac{\bar{F}_{Y \mid X=x_{1}}(y)}{\bar{F}_{Y \mid X=x_{2}}(y)} \leq \frac{1}{\pi_{n_{x_{2}} x_{2}}} \frac{F_{U \mid X=x_{1}}\left(r^{\mathrm{e}}\left(y, x_{1}\right)\right)}{F_{U \mid X=x_{2}}\left(r^{\mathrm{e}}\left(y, x_{2}\right)\right)},
$$

for all $y \geq y^{\prime}$. By Step 2, there exists $\tilde{y} \in \mathbb{R}$ such that $r^{\mathrm{e}}\left(y, x_{1}\right) \leq \lambda r^{\mathrm{e}}\left(y, x_{2}\right)$ for all $y \geq \tilde{y}$. Therefore, for all $y \geq \max \left\{\tilde{y}, y^{\prime}\right\}$,

$$
\frac{F_{U \mid X=x_{1}}\left(r^{\mathrm{e}}\left(y, x_{1}\right)\right)}{F_{U \mid X=x_{2}}\left(r^{\mathrm{e}}\left(y, x_{2}\right)\right)} \leq \frac{F_{U \mid X=x_{1}}\left(\lambda_{1} r^{\mathrm{e}}\left(y, x_{2}\right)\right)}{F_{U \mid X=x_{2}}\left(r^{\mathrm{e}}\left(y, x_{2}\right)\right)}=\frac{F_{U \mid X=x_{1}}\left(\lambda_{1} r^{\mathrm{e}}\left(y, x_{2}\right)\right)}{F_{U \mid X=x_{1}}\left(r^{\mathrm{e}}\left(y, x_{2}\right)\right)} \frac{F_{U \mid X=x_{1}}\left(r^{\mathrm{e}}\left(y, x_{2}\right)\right)}{F_{U \mid X=x_{2}}\left(r^{\mathrm{e}}\left(y, x_{2}\right)\right)}
$$

The first term on the right hand side of (1.3) converges to 0 as $y \rightarrow+\infty$ by S3 (ii), and the second term on the right hand side of (1.3) is bounded by S3 (iii). Combining this result with (1.2), we obtain the conclusion.

Proof of (ii). From Part (i), it is sufficient to show that S3 (i) implies S3 (i'). Pick any $\epsilon>0$. By S3 (i), there exists $\bar{y} \in \mathbb{R}$ such that $\left[r\left(y, x_{1}\right) / r\left(y, x_{2}\right)\right] \in(\lambda-\epsilon / 2, \lambda+\epsilon / 2)$ for all $y \geq \bar{y}$. This implies $\inf _{y \geq \bar{y}}\left[r\left(y, x_{1}\right) / r\left(y, x_{2}\right)\right] \in(\lambda-\epsilon, \lambda+\epsilon)$ for all $y \geq \bar{y}$. Therefore, S3 (i') is guaranteed.

Proof of (iii). By Proposition 1 and Lemma 1 of EK,

$$
\lim _{y \rightarrow+\infty} \frac{\bar{F}_{Y \mid X=x_{1}}(y)}{\bar{F}_{Y \mid X=x_{2}}(y)} \leq \lim _{y \rightarrow+\infty} \frac{1}{\pi_{n_{x_{2} x_{2}}}} \frac{F_{U \mid X=x_{1}}\left(r^{\mathrm{e}}\left(y, x_{1}\right)\right)}{F_{U \mid X=x_{2}}\left(r^{\mathrm{e}}\left(y, x_{2}\right)\right)} \leq \lim _{y \rightarrow+\infty} \frac{1}{\pi_{n_{x_{2} x_{2}}}} \frac{F_{U \mid X=x_{1}}\left(r^{\mathrm{e}}\left(y, x_{2}\right)\right)}{F_{U \mid X=x_{2}}\left(r^{\mathrm{e}}\left(y, x_{2}\right)\right)}
$$

where the last inequality follows from $\mathrm{S} 2$, which implies $r^{\mathrm{e}}\left(y, x_{1}\right) \leq r^{\mathrm{e}}\left(y, x_{2}\right)$. Therefore, S1 (ii) and S3 (iii) imply the conclusion.

Proof of (iv). We first argue that S1 and S3 (i') are not sufficient for S2. Let $x_{1}=1, x_{2}=3$, and

$$
r(y, x)=\left\{\begin{array}{ll}
-y^{x}-x & \text { for } y \in(-\infty, 0) \\
-y^{\frac{1}{x}}-x & \text { for } y \in[0,+\infty)
\end{array} .\right.
$$

Then $r(y, x)$ satisfies S1. Also, since $\inf _{y^{\prime} \geq y}\left[r\left(y^{\prime}, x_{1}\right) / r\left(y^{\prime}, x_{2}\right)\right]=r\left(y, x_{1}\right) / r\left(y, x_{2}\right)$ in this case, L'Hôpital's rule implies that S3 (i') is satisfied with $\lambda=+\infty$. On the other hand, from $r(0,1)>r(0,3), \mathrm{S} 2$ is not satisfied.

\footnotetext{
${ }^{1}$ Note that $\mathrm{S} 1$ is sufficient for Proposition 1 and Lemma 1 of EK.
} 
We next argue that S1 and S3 (i') are not necessary for S2. Let $x_{1}=0, x_{2}=1$, and

$$
r(y, x)=-y+\left(\frac{x}{1+y^{2}}\right) .
$$

Then $r(y, x)$ satisfies S1. and S2. Also, since $\inf _{y^{\prime} \geq y}\left[r\left(y^{\prime}, x_{1}\right) / r\left(y^{\prime}, x_{2}\right)\right]=r\left(y, x_{1}\right) / r\left(y, x_{2}\right)$ in this case, L'Hôpital's rule implies $\lim _{y \rightarrow+\infty} \inf _{y^{\prime} \geq y}\left[r\left(y^{\prime}, x_{1}\right) / r\left(y^{\prime}, x_{2}\right)\right]=1$. Thus, S3 (i') is not satisfied.

Proof of (v). We first show sufficiency. In the proof of Part (i), we have shown that under $\mathrm{S} 1$ and $\mathrm{S} 3$ (i'), for any $\lambda_{1} \in(1, \lambda)$, there exists $\bar{y} \in \mathbb{R}$ such that $r\left(y, x_{1}\right) \leq \lambda_{1} r\left(y, x_{2}\right)$ and $r\left(y, x_{2}\right) \leq-1$ for all $y \geq \bar{y}$. Therefore, $r\left(y, x_{1}\right) \leq \lambda_{1} r\left(y, x_{2}\right)<r\left(y, x_{2}\right)$ for all $y \geq \bar{y}$ and the sufficiency is obtained.

We next argue that S1 and complementarity at extremes do not necessarily imply S3(i). Note that S2 implies complementarity at extremes. Therefore, the second example of the proof of Part (iv) guarantees the conclusion.

Proof of (vi). In the proof of proposition (i), we use S3 (i') only to derive the fact that there are $\lambda_{1}>1$ and $y_{o}$ such that $\lambda_{1} r^{\mathrm{e}}\left(y, x_{2}\right)>r^{\mathrm{e}}\left(y, x_{1}\right)$ for all $y \geq y_{o}$. Thus, it is enough to find functions $r\left(y, x_{1}\right)$ and $r\left(y, x_{2}\right)$ of $y$ that satisfy S1 and $\lambda_{1} r^{\mathrm{e}}\left(y, x_{2}\right)>r^{\mathrm{e}}\left(y, x_{1}\right)$ for large enough $y$ but do not satisfy S3(i'). Let

$$
r\left(y, x_{1}\right)=-\frac{3}{2} y
$$

For $r\left(y, x_{2}\right)$, we take some constant $c>0$ and define sequences $\left\{a_{j}\right\}_{j \in \mathbb{N}},\left\{b_{j}\right\}_{j \in \mathbb{N}}$, and $\left\{d_{j}\right\}_{j \in \mathbb{N}}$ as

$$
\begin{gathered}
a_{j}=\left\{\begin{array}{cl}
-3 & \text { if } j \text { is odd } \\
1 & \text { if } j \text { is even }
\end{array}\right. \\
b_{1}=c, \quad b_{2}=3 c, \quad b_{j}=7 b_{j-2} \text { for } j \geq 3, \\
d_{0}=-\infty, \quad d_{1}=c, \quad d_{2}=2.5 c, \quad d_{j}=7 d_{j-2} \text { for } j \geq 3 .
\end{gathered}
$$

Then we define

$$
r\left(y, x_{2}\right)=a_{j} y+b_{j}
$$

for $y \in\left(d_{j-1}, d_{j}\right]$ and $j \in \mathbb{N}$. Observe that $r\left(y, x_{1}\right)$ and $r\left(y, x_{2}\right)$ satisfy $\mathrm{S} 1$. Moreover,

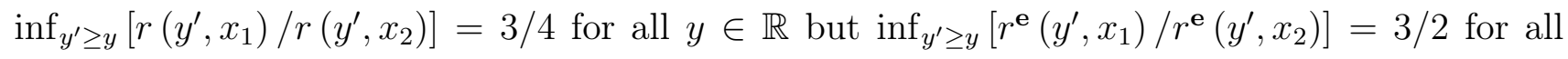
$y \in \mathbb{R}$.

Remarks. 1. Proposition (i) derives the conclusion of Theorem 1 of EK without using S2. Indeed S3 (i') plays a role of complementarity in the tails, which guarantees the inequality in (1.1). Since S3 (i') implies S3 (i), we can argue that the conclusion of Theorem 1 can be derived by only S1 and S3. This point is presented in Proposition (ii). Therefore, monotonicity of the conditional distribution $F_{Y \mid X=x}$ in $x$ in the tails may not be a testable implication of S2. On the 
other hand, if we impose S2 instead of S3 (i), the inequality in (1.1) can be guaranteed only for $\lambda=1$. Therefore, under S2 without S3 (i), we can only say that the ratio $\bar{F}_{Y \mid X=x_{1}}(y) / \bar{F}_{Y \mid X=x_{2}}(y)$ does not diverge as $y \rightarrow+\infty$. This point is presented in Proposition (iii).

2. Proposition (iv) clarifies that there is no logical relationship for the two complementary conditions, S2 and S3 (i'). Therefore, under S1, the rejection of the testable implication of Theorem 1 implies the rejection of S3 (i') but does not necessarily imply the rejection of S2. Proposition (v) compares S3 (i') and a weaker notion on S2, complementarity at extremes. This notion is weak enough so that S3 (i') implies complementarity at extremes, but not vice versa. Therefore, the rejection of the testable implication of Theorem 1 does not necessarily imply the rejection of complementarity at extremes. Since S3 (i) implies S3 (i'), the above arguments remain true when we replace S3 (i') with S3 (i).

3. A key insight from the above argument is that we need a stronger notion of complementarity so that the ratio $r\left(y, x_{1}\right) / r\left(y, x_{2}\right)$ is bounded away from 1 at least in the tails. For example, we can strengthen $\mathrm{S} 2$ by the following one.

Assumption S2'. $r(y, x)$ is monotone increasing in $x$ on $\mathbb{R}$. Furthermore, as $y \rightarrow+\infty$, $r\left(y, x_{1}\right) / r\left(y, x_{2}\right)$ neither converges to 1 nor contacts to 1 infinitely many times.

Then since S2' implies S3 (i'), Proposition (i) guarantees that under S1, S2', and S3 (ii)-(iii), the conclusion of Theorem 1 holds true. We summarize this point in the following corollary.

Corollary. Under S1, S2', and S3 (ii)-(iii), the conclusion of Theorem 1 holds true.

4. Proposition (vi) considers the meaning of acceptance of the testable implication. This result implies that the acceptance of the testable implication in Theorem 1 does not necessarily imply the acceptance of complementarity at extreme (and S2 as well).

5. EK considered an alternative set of assumptions for S3:

Assumption S3'. (For any $x_{1}, x_{2} \in \mathcal{X}$ with $x_{1}<x_{2}$ ) (i) $\lim _{y \rightarrow+\infty}\left[r\left(y, x_{1}\right)-r\left(y, x_{2}\right)\right]=\delta$ with $\delta<0$; (ii) for any $\delta<0, \lim _{u \rightarrow-\infty} F_{U \mid X=x_{1}}(u+\delta) / F_{U \mid X=x_{1}}(u)=0$; (iii) $F_{U \mid X=x_{1}}(u) / F_{U \mid X=x_{2}}(u)$ is bounded as $u \rightarrow-\infty$.

We can derive an analogous proposition and remarks if we replace S3 (i') with the following assumption.

Assumption S3'. (i') $\lim _{y \rightarrow+\infty} \inf _{y^{\prime} \geq y}\left[r\left(y^{\prime}, x_{1}\right)-r\left(y^{\prime}, x_{2}\right)\right]=\delta$ with $\delta<0$.

\section{REFERENCES}

[1] Echenique, F. and I. Komunjer (2009) Testing models with multiple equilibria by quantile methods, Econometrica, 77, 1281-1297. 
Faculty of Economics, Keio University, 2-15-45 Mita, Minato-Ku, Tokyo 108-8345, Japan.

E-mail address: yasur88@a2.keio.jp

Department of Economics, London School of Economics and Political Science, Houghton Street, London WC2A 2AE, UK.

E-mail address: t.otsu@lse.ac.uk 